\title{
Algorithms for locating extremely conserved elements in multiple sequence alignments Huei-Hun E Tseng ${ }^{1}$ and Martin Tompa* ${ }^{1,2}$
}

\author{
Addresses: ${ }^{1}$ Department of Computer Science and Engineering, University of Washington, Box 352350, Seattle, WA, 98195-2350, USA and \\ ${ }^{2}$ Department of Genome Sciences, University of Washington, Seattle, WA, 98195-5065, USA \\ E-mail: Huei-Hun E Tseng - lachesis@cs.washington.edu; Martin Tompa* - tompa@cs.washington.edu \\ ${ }^{*}$ Corresponding author
}

Published: 18 December 2009

BMC Bioinformatics 2009, 10:432 doi: 10.1/86/1471-2105-10-432

This article is available from: http://www.biomedcentral.com/I47/-2/05/I0/432

(c) 2009 Tseng and Tompa; licensee BioMed Central Ltd.

This is an Open Access article distributed under the terms of the Creative Commons Attribution License (http://creativecommons.org/licenses/by/2.0), which permits unrestricted use, distribution, and reproduction in any medium, provided the original work is properly cited.
Received: 29 July 2009

Accepted: 18 December 2009

\begin{abstract}
Background: In 2004, Bejerano et al. announced the startling discovery of hundreds of "ultraconserved elements", long genomic sequences perfectly conserved across human, mouse, and rat. Their announcement stimulated a flurry of subsequent research.

Results: We generalize the notion of ultraconserved element in a natural way from extraordinary human-rodent conservation to extraordinary conservation over an arbitrary set of species. We call these "Extremely Conserved Elements". There is a linear time algorithm to find all such Extremely Conserved Elements in any multiple sequence alignment, provided that the conservation is required to be across all the aligned species. For the general case of conservation across an arbitrary subset of the aligned species, we show that the question of whether there exists an Extremely Conserved Element is NP-complete. We illustrate the linear time algorithm by cataloguing all 177 Extremely Conserved Elements in the currently available 44-vertebrate wholegenome alignment, and point out some of the characteristics of these elements.

Conclusions: The NP-completeness in the case of conservation across an arbitrary subset of the aligned species implies that it is unlikely an efficient algorithm exists for this general case. Despite this fact, for the interesting case of conservation across all or most of the aligned species, our algorithm is efficient enough to be practical. The 177 Extremely Conserved Elements that we catalog demonstrate many of the characteristics of the original ultraconserved elements of Bejerano et al.
\end{abstract}

\section{Background}

In 2004, Bejerano et al. [1] made the startling discovery that there are hundreds of long genomic sequences extraordinarily conserved across human, mouse, and rat, most of them in noncoding regions and some of them very distant from the nearest human gene. They defined an "ultraconserved element" to be at least 200 consecutive alignment columns, $100 \%$ of these columns perfectly conserved in human, mouse, and rat. They reported 481 such elements across the human genome, exclusive of rRNA genes. They also reported incidentally that some fraction $(99 \%, 97 \%$, and $67 \%$, respectively) of these ultraconserved elements are also well conserved in $\mathrm{dog}$, in chicken, and in fugu, though with fewer than $100 \%$ of the columns perfectly conserved in these other species even in this fraction of elements.

Their introduction of ultraconserved elements stimulated a flurry of subsequent research. Derti et al. [2] compiled lists of similarly perfectly conserved elements in human- 
mouse-dog and in human-chicken, and observed a surprisingly small overlap in these three sets of ultraconserved elements. Sakuraba et al. [3] compiled a list of elements each more than 500 bp long and each more than 95\% identical between human and mouse, and observed that only $9-14 \%$ of these have conserved matches in fishes. Visel et al. [4] showed that the noncoding ultraconserved elements of Bejerano et al. [1] were only a subset of elements under similar constraint and with similar regulatory function. They generated this larger set of elements using Gumby scores [5], and showed that this larger set of noncoding ultraconserved elements is highly enriched in mouse enhancer activity. Rather than using percent of perfectly conserved columns, Siepel et al. [6] incorporated the phylogeny into the measure of conservation by using a phylogenetic hidden Markov model, and defined Highly Conserved Elements to be those with the highest log-odds scores, measuring how much more likely they are to be generated in the conserved than the nonconserved state of the model.

Most of these works defined their conserved elements in terms of conservation across just 2 or 3 species, Siepel et al. [6] being an exception. In this paper we extend the notion of ultraconserved elements in a very natural way to an arbitrarily large collection of species.

Suppose that you are given a whole-genome multiple sequence alignment, such as the current 44-vertebrate whole-genome alignment available through the UCSC Genome Browser [7]. You are interested in finding long regions of this alignment that are extraordinarily well conserved across all or most of the 44 species, in the spirit of generalizing the notion of ultraconserved elements of Bejerano et al. [1]. For concreteness in this introduction, let us say that you want to identify all regions of at least 100 consecutive alignment columns such that, for some subset $S$ of at least 40 of the 44 species, at least $80 \%$ of the columns in this region are perfectly conserved (i.e., contain the same nucleotide) across all the species in $S$. Because the 44-vertebrate whole-genome alignment occupies approximately 250 gigabytes of memory, algorithmic efficiency is a concern.

In the next section, we consider the generalization of this problem instance to arbitrary alignments, any number of columns, any minimum cardinality $|S|$, and any percentage of conserved columns. We call such well conserved regions "Extremely Conserved Elements". We present a linear time algorithm for finding all such elements, provided the subset $S$ is the entire set of aligned species. For an arbitrary subset $S$, we demonstrate that the problem is NP-complete [8] and hence it is unlikely that there is an efficient algorithm for finding Extremely Conserved Elements in this general case.
As an illustration of the linear time algorithm, we present results for the concrete instantiation of the problem given above. In the current 44 -vertebrate wholegenome alignment, there are 177 elements, each with at least 100 alignment columns and each perfectly conserved across the same 40 or more vertebrates in at least $80 \%$ of its columns. The longest such element is 355 columns long and occurs $60 \mathrm{Kbp}$ from the nearest gene on human chromosome 19, perfectly conserved in $80 \%$ of its columns across 41 of the species, missing only gorilla, shrew, and lamprey. There is also a region $70 \mathrm{bp}$ long, $7 \mathrm{Kbp}$ upstream of the FOXB2 gene on human chromosome 9, with $90 \%$ of its columns perfectly conserved across all 44 vertebrates.

\section{Results and discussion \\ Problem statement and algorithms}

We begin with a precise formulation of the Extremely Conserved Element problem:

Inputs: $m \times n$ alignment matrix $M$ with entries from $\{\mathrm{A}$, $\mathrm{C}, \mathrm{G}, \mathrm{T},-\}$, integer $s \leq m$, integer $t \leq n$, and real number $0<c \leq 1$.

Problem: Determine if $M$ has a subset $S$ of rows, a subset $T$ of consecutive columns (ignoring columns that contain the gap character "-" in every row of $S$ ), and a subset $U$ of $T$, with $|S| \geq s,|T| \geq t$, and $|U| \geq c|T|$ such that, in the matrix $M$ restricted to $S \times U$, every column is perfectly conserved (that is, all the elements in that column are equal). Note that neither $S$ nor $U$ need be consecutive.

For example, the illustrative problem in the introduction is the version of the Extremely Conserved Element problem with $m=44, n \approx 3.8 \times 10^{9}, s=40, t=100$, and $c=0.8$.

The condition that every column in $S \times U$ be perfectly conserved is overly simplistic. More realistically, the conservation measure should depend on the phylogeny relating the species, as does, for example, the measure used by phastCons [6]. Suppose that $F$ is any conservation scoring function (that may depend on the phylogeny). The algorithm of Theorem 1 is easily generalized to the problem in which every column in $S \times U$ must have $F$ exceeding some threshold $\tau$.

Theorem 1: If $s=m$, the Extremely Conserved Element problem can be solved in time $\mathrm{O}(\mathrm{mn})$. In fact, the maximum value of $t$ can be determined in this time.

Proof: Assume without loss of generality that no column contains the gap character "-" in every row. For $1 \leq i \leq n$, 
let $q_{i}=1$ if column $i$ is perfectly conserved, and $q_{i}=0$ otherwise. The result then follows from Theorem 2.

Theorem 2: Given $q \in\{0,1\}^{n}$ and $0<c \leq 1$, there is an $\mathrm{O}$ $(n)$ time algorithm that maximizes $j-i$ subject to the condition that $q_{i+1} \ldots q_{j}$ contains at least $c(j-i) 1^{\prime}$ s.

Proof: A variety of linear time algorithms for this problem have appeared in the literature [9-11], although this is the first time it has been applied to multiple sequence alignments on a gigabyte scale. We give here a new and simpler algorithm due to Eddie Grove and Benno Schwikowski (personal communication). For $0 \leq i$ $\leq n$, let

$$
r_{i}=\sum_{k=1}^{i}\left(q_{k}-c\right)
$$

Let $x$ be the number of 1 's in $q_{i+1} \ldots q_{j}$. Then

$$
r_{j}-r_{i}=\sum_{k=i+1}^{j}\left(q_{k}-c\right)=x(1-c)+(j-i-x)(-c)=x-c(j-i) .
$$

Thus, $r_{j} \geq r_{i}$ if and only if $q_{i+1} \ldots q_{j}$ has at least $c(j-i) 1$ 's, so the objective is to maximize $j-i$ subject to $r_{j} \geq r_{i}$.

For $0 \leq i \leq n$, let $X_{i}=\min \left(r_{0}, r_{1}, \ldots, r_{i}\right)$ and $Y_{i}=\max \left(r_{i}\right.$ $\left.r_{i+1}, \ldots, r_{n}\right) . X$ and $\mathrm{Y}$ are each nonincreasing sequences. We claim that the objective above is equivalent to maximizing $j$ - $i$ subject to $Y_{j} \geq X_{i}$ : If $r_{j} \geq r_{i}$, then $Y_{j}=\max$ $\left(r_{j}, r_{j+1}, \ldots, r_{n}\right) \geq r_{j} \geq r_{i} \geq \min \left(r_{0}, r_{1}, \ldots, r_{i}\right)=X_{i}$. If $Y_{j} \geq X_{i}$ and $X_{i}<X_{i-1}$ and $Y_{j}>Y_{j+1}$, then $r_{i}=X_{i}$ and $r_{j}=Y_{j}$, so $r_{j} \geq r_{i}$. In particular, $(i, j)$ maximizes $j-i$ subject to $r_{j} \geq r_{i}$ if and only if $(i, j)$ maximizes $j-i$ subject to $Y_{j} \geq X_{i}$. Since $X$ and $Y$ are sorted, the latter can be found in linear time: merge $X$ and $Y$, breaking ties by taking elements from $Y$ first. Then identify the maximum $j-i$ such that $Y_{j}$ and $X_{i}$ are adjacent (in this order) in the sorted list. $\quad \square$

In fact, in the proof of Theorem 2, any pair $(i, j)$ such that $Y_{j}$ and $X_{i}$ are adjacent (in this order) in the sorted list corresponds to a maximal interval $q_{i+1} \ldots q_{j}$ that contains at least $c(j-i) 1$ 's. Thus, all such maximal intervals can be found in linear time.

The dual of Theorem 2, maximizing $c$ subject to a lower bound on $j-i$, also can be accomplished in linear time $[12,13]$. This implies that, for $s=m$, the maximum value of $c$ in the Extremely Conserved Element problem can also be determined in time $\mathrm{O}(\mathrm{mn})$.

For arbitrary $S$, the following theorem provides another special case in which the problem can be solved efficiently.
Theorem 3: If $c=1$, the Extremely Conserved Element problem can be solved in polynomial time. In fact, the maximum value of $s$ can be determined in this time.

Proof: For every choice $T$ of at least $t$ consecutive columns, sort the rows of $T$ lexicographically and look for $s$ identical rows, with at least $t$ nongap characters each, in this sorted list.

However, for the general case, the following theorem shows that it is unlikely that there is an efficient algorithm [8].

Theorem 4: The general Extremely Conserved Element problem is NP-complete, even if $t=n$ and $M^{\prime}$ s entries are all either $\mathrm{A}$ or $\mathrm{T}$.

Proof: The reduction used is very similar to the reduction from Clique to Balanced Complete Bipartite Subgraph [14].

Let $(G, K)$ be an instance of the Clique problem, where $G=(V, E)$ is an undirected graph and $K$ is an integer. Assume without loss of generality that $K<|V|-2$. Consider the bipartite graph $B=((X, Y), F)$, where $X=V$, $Y=E$, and $F=\{(v, e) \mid v \notin e\}$. Let $M$ be the $|X| \times|Y|$ adjacency matrix of $B$, where $M_{i j}=\mathrm{A}$ if $\left(X_{i}, Y_{j}\right) \in F$ and $M_{i j}=$ T otherwise. Let $s=|V|-K, t=|E|$, and $c=\left(\begin{array}{c}K \\ 2\end{array}\right) /|E|$. We will show that $G$ has a $K$-clique if and only if $M$ has the appropriate Extremely Conserved Element.

Suppose that $C \subseteq V$ is a $K$-clique of $G$. Choose $S=V-C$ and $U=\{\{u, v\} \mid u \in C$ and $v \in C\} .|S|=|V|-K$ and $|U|=$ $\left(\begin{array}{l}K \\ 2\end{array}\right)$ and, in $B$, every vertex in $S$ is adjacent to every vertex in $U$. Hence, the submatrix of $M$ restricted to $S \times U$ contains only the character A.

Conversely, suppose that $M$ has a $(|V|-K) \times\left(\begin{array}{l}K \\ 2\end{array}\right)$ submatrix $M^{\prime}$ each of whose columns is perfectly conserved. Since each vertex $v$ in $Y$ is not adjacent to exactly 2 vertices in $X$, it is impossible for $v^{\prime}$ s column of $M^{\prime}$ to consist only of the character T when $|V|-K>2$, as is the case. Hence, $M^{\prime}$ contains only the character $A$. That is, there are subsets $S$ of $X$ and $U$ of $Y$ that form a complete bipartite subgraph of $B$, with $|S|=|V|-K$ and $|U|=\left(\begin{array}{l}K \\ 2\end{array}\right)$. Every vertex $\{u, v\} \in U$ must satisfy $u \in V-S$ and $v \in V-S$ since, in $B,\{u, v\}$ is adjacent to every vertex 
in $S$. But $|V-S|=K$, so the $\left(\begin{array}{l}K \\ 2\end{array}\right)$ vertices in $U$ correspond to the edges of a K-clique $V-S$ in $G$. $\quad \square$

\section{Catalog of Extremely Conserved Elements}

As an illustration of Theorem 1, we present results for the concrete instantiation of the problem given in the introduction. In the current 44 -vertebrate whole-genome MULTIZ alignment (human genome assembly UCSC hg18, March 2006) available through the UCSC Genome Browser [7], we identify all Extremely Conserved Elements with at least 100 consecutive alignment columns such that, for some subset $S$ of at least 40 species including human, at least $80 \%$ of the columns in this region are perfectly conserved across all the species in $S$. (If some column contains the gap character "_" in all species of $S$, that column is ignored, contributing neither to the count of 100 columns nor to the percent perfectly conserved.) We refer to such elements as $E C(40,100,0.8)$ elements.

Despite the NP-completeness demonstrated in Theorem 4, the algorithm of Theorem 1 still allows a feasible solution to this particular instantiation of the general Extremely Conserved Element problem. The simple reason is that $\sum_{s=40}^{44}\left(\begin{array}{c}44 \\ s\end{array}\right)=149,986$ is not an impossibly large number of combinations on which to run the linear time algorithm. Had we asked for conservation across only 22 rather than 40 species, the computation would have been prohibitive, because $\left(\begin{array}{l}44 \\ 22\end{array}\right)>2 \times 10^{12}$. In addition, there are two novel filters described in Methods that make the application to whole-genome alignments feasible.

In the current 44-vertebrate whole-genome alignment, our algorithm produces $177 \mathrm{EC}(40,100,0.8)$ elements. A complete listing of these elements is given in additional files. Additional file 1 is a spreadsheet that shows each EC $(40,100,0.8)$ element's human genome coordinates, its length, percent identity, names of missing species, name of the human gene in which it resides (if appropriate), names of and distances to the two nearest neighboring genes, and overlap with previously identified conserved elements $[1,4,6]$. Additional file 2 shows the exact alignment for each $\operatorname{EC}(40$, $100,0.8$ ) element. Additional file 3 shows the single longest extremely conserved element that has at least $90 \%$ of its columns perfectly conserved across all 44 vertebrate species. It is $70 \mathrm{bp}$ long. (In Additional files 1 and 2, the lengths of some of the elements are shown as slightly less than 100 columns. The reason is that we
Table I: Enrichment for biological process GO terms of the $\mathbf{2 4 0}$ human genes containing or neighboring $\operatorname{EC}(40,100,0.8)$ elements

\begin{tabular}{lccc}
\hline GO category & $\begin{array}{c}\text { observed } \\
\text { genes }\end{array}$ & $\begin{array}{c}\text { expected } \\
\text { number }\end{array}$ & p-value \\
\hline regulation of transcription & 70 & 25.68 & $3.30 \times 10^{-17}$ \\
development & 43 & 22.36 & $1.04 \times 10^{-5}$ \\
organ development & 18 & 6.74 & $1.34 \times 10^{-4}$ \\
nervous system development & 16 & 5.62 & $1.56 \times 10^{-4}$ \\
\hline
\end{tabular}

have removed from every element those columns at each end that are not perfectly conserved. Any of these elements can be padded on either end to 100 columns while still maintaining at least $80 \%$ identical columns.)

For the 240 human genes either containing an $\operatorname{EC}(40$, $100,0.8$ ) element, or immediately upstream or downstream from that element if it is between genes, we investigated enrichment for biological process GO terms, using WebGestalt [15]. The results are shown in Table 1. These enrichments are in accord with previous humanrodent studies $[1,4]$, which reported significant functional enrichment for genes involved in regulation of transcription, DNA binding, development, and nervous system development.

For the genes containing the $25 \mathrm{EC}(40,100,0.8)$ elements that overlap human coding exons, there is functional enrichment for RNA processing $\left(p\right.$-value $\left.=2.20 \times 10^{-4}\right)$. This is consistent with the reported findings for genes containing ultraconserved elements that overlap coding exons, which show significant functional enrichment for RNA binding and regulation of splicing [1].

Figure 1 shows the distribution of the $177 \mathrm{EC}(40,100$, 0.8 ) elements by human chromosome and location with respect to human genes. If an element overlaps a human coding exon (in some splice form), it is called "partially coding". If not and it is entirely contained within an intron between two coding exons (in some splice form), it is called "intronic". If it is between the annotated transcription start and stop sites of a gene but is neither partially coding nor intronic, it is called "UTR". (Most of these elements are actually contained in a UTR intron rather than overlapping the mature UTR.) If it is entirely contained between two genes (in all splice forms), it is called "intergenic". In total there are 25 partially coding, 14 UTR, 61 intronic, and 77 intergenic $\operatorname{EC}(40,100,0.8)$ elements; these account for $14 \%, 8 \%, 34 \%$, and $44 \%$, respectively, of all 177 elements. None of the elements overlap annotated rRNA, tRNA, or other annotated noncoding RNA genes. Of the 77 intergenic elements, 65 (37\% of all 177 elements) are more than $10 \mathrm{Kbp}$ from the nearest human gene and 42 (24\% of all 
Distribution by Chromosome and Location

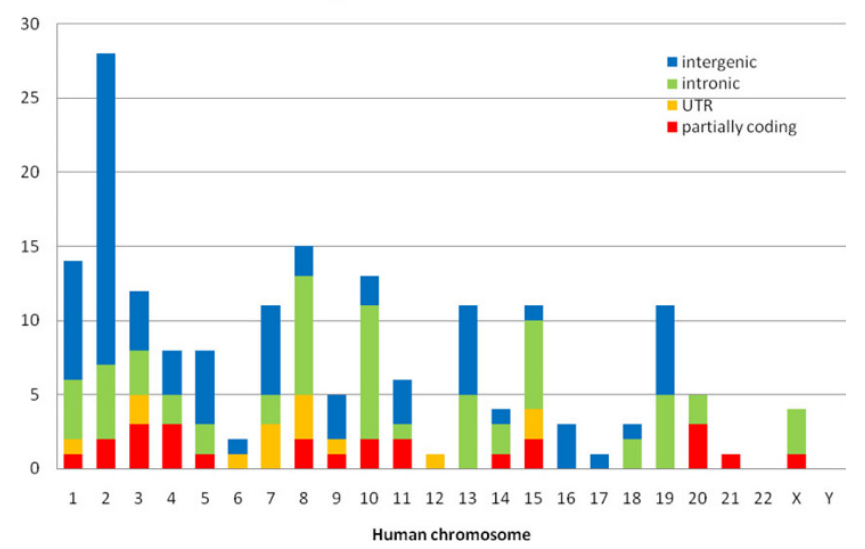

Figure I

Distribution of $177 \mathrm{EC}(40,100,0.8)$ elements by human chromosome and location with respect to human genes. See text for the explanation of location labels.

177 elements) are more than $100 \mathrm{Kbp}$ from the nearest gene. These percentages are somewhat greater than the corresponding percentages (29\% over $10 \mathrm{Kbp}$ and $18 \%$ over $100 \mathrm{Kbp}$ ) for intergenic ultraconserved elements [1].

As was observed for ultraconserved elements [1], it is quite common to see three to eight $\operatorname{EC}(40,100,0.8)$ elements clustered within the same gene or within the same intergenic region (see Additional file 1). As one extreme example, eight occur within the introns and another two upstream of the human gene ZFPM2 on chromosome 8. ZFPM2 is a zinc finger transcription factor that modulates the activity of GATA family proteins, which are important regulators of cardiogenesis. None of these ten ZFPM2 EC $(40,100,0.8)$ elements overlap ultraconserved elements of Bejerano et al. [1], and three of them do not even overlap the larger set of constrained human-rodent elements of Visel et al. [4], so are novel.

The longest $\mathrm{EC}(40,100,0.8)$ element found is 355 columns long and is perfectly conserved in $80 \%$ of its columns across 41 of the species, missing only gorilla, shrew, and lamprey. It occurs $60 \mathrm{Kbp}$ downstream of the nearest gene on human chromosome 19. But interestingly it is $297 \mathrm{Kbp}$ upstream of its other neighboring gene ZNF536, which has two other upstream and three intronic EC $(40,100,0.8)$ elements. ZNF536 is a zinc finger protein, expressed in the developing central nervous system, that negatively regulates neuron differentiation [16]. Only two of these six EC(40, 100, 0.8) elements overlap ultraconserved elements of Bejerano et al. [1].
Note, though, that the distribution of the lengths of EC $(40,100,0.8)$ elements is not significant since, as pointed out in Methods, our algorithm sacrifices length in preference for number of species in the element. As one extreme example, there are abutting elements at human hg1 8 coordinates chrX:24826152-24826332 and chrX:24826333-24826597 of lengths 188 and 276, respectively. The first is missing alpaca, sloth, medaka, and lamprey, while the second is missing only alpaca, medaka, and lamprey. Had the algorithm not prioritized number of species over length, it would have produced a single EC(40, 100, 0.8) element of length 464, which would have been longer than the current longest element. These two current elements are two out of three $\operatorname{EC}(40,100,0.8)$ elements that occur in an intron of POLA1, which encodes the DNA polymerase alpha catalytic subunit.

Lamprey, the species most distant from the mammals and also a low-coverage genome assembly, is missing from 170 of the $177 \mathrm{EC}(40,100,0.8)$ elements. The species with the next highest numbers are gorilla (missing from 41), cat (missing from 35), and zebrafish (missing from 30). Like lamprey, gorilla and cat are lowcoverage genome assemblies. On the other hand, fugu is missing from only 4 of the $177 \mathrm{EC}(40,100,0.8)$ elements, zebra finch is missing from only 3, chicken is missing from only 2 , and lizard is missing from only 1. These figures suggest that depth of sequencing is a greater determinant of inclusion in extremely conserved elements than is evolutionary distance, and also suggest that additional extremely conserved elements will emerge as the quality of genome sequences increases.

$47(27 \%)$ of the $177 \mathrm{EC}(40,100,0.8)$ elements overlap an ultraconserved element of Bejerano et al. [1]. 117 $(66 \%)$ overlap the larger set of constrained humanrodent elements of Visel et al. [4]. These overlaps confirm the fact that we have identified significant conserved elements that cannot be found by applying a more stringent conservation criterion to a smaller collection of species such as human and rodents. All 177 EC(40, 100, 0.8) elements overlap phastCons Highly Conserved Elements [6] (as computed in the UCSC 44-vertebrate alignment) with phastCons logodds scores each exceeding 1300. Details of all these overlaps are shown in Additional file 1. The phastCons Highly Conserved Elements have the realistic advantage of taking the phylogeny and branch lengths into account, which our Extremely Conserved Elements do not. However, because their log-odds scores favor longer alignments, phastCons Highly Conserved Elements tend to be much longer than $\mathrm{EC}(40,100,0.8)$ elements, with less intense concentration of extremely conserved columns. There are 12,749 phastCons Highly Conserved 
Elements with log-odds score at least 1300, of which only a certain 177 contain within them $\operatorname{EC}(40,100,0.8)$ elements. The average length of these 12,749 elements is $500 \mathrm{bp}$, with many of them several kilobasepairs long, whereas the average $\operatorname{EC}(40,100,0.8)$ element is less than $133 \mathrm{bp}$ long. There is no obvious way of querying phastCons Highly Conserved Elements so as to identify those with such intense concentration of extremely conserved columns. For instance, if you restrict attention to those Highly Conserved Elements with log-odds score at least 1300 and length at most $400 \mathrm{bp}$, this long list would contain only 43 of the $177 \operatorname{EC}(40,100,0.8)$ elements; the remaining 134, though as extremely conserved, occur within longer Highly Conserved Elements.

Of the genes containing $\operatorname{EC}(40,100,0.8)$ elements that do not contain nor are adjacent to either ultraconserved elements of Bejerano et al. [1] nor the larger set of constrained human-rodent elements of Visel et al. [4], three are particularly notable because their functions are closely related to the GO term enrichments discussed near the beginning of this section. NLGN1 on human chromosome 3 contains an intronic EC(40, 100, 0.8) element and is involved in nervous system development. In particular, this gene encodes a member of a family of neuronal cell surface proteins that may be involved in the formation and remodeling of central nervous system synapses. WHSC1L1 on human chromosome 8 contains a partially coding $\mathrm{EC}(40,100,0.8)$ element and is involved both in regulation of transcription and in cell differentiation. More specifically, this gene encodes a histone methyltransferase that preferentially methylates K4 and K27 of histone H3, which are epigenetic tags for transcriptional regulation. RBM5 on human chromosome 3 contains a partially coding $\mathrm{EC}(40,100,0.8)$ element and, like many other genes with such coding elements, is involved in RNA processing. In particular, this gene encodes a component of the spliceosome A complex and regulates alternative splicing of a number of mRNAs, including FAS and CASP2/caspase-2 in the apoptosis process. In the case of FAS, it promotes exclusion of exon 6, producing a soluble form of FAS that inhibits apoptosis. In the case of CASP2/caspase-2, it promotes exclusion of exon 9, producing a catalytically active form of CASP2/Caspase-2 that induces apoptosis. These three genes, NLGN1, WHSC1L1, and RBM5, are just some of the genes containing extremely conserved elements that were not uncovered in previous studies of ultraconservation.

\section{Conclusions}

The notion of ultraconserved element was introduced by Bejerano et al. [1] to point out that there are certain long genomic sequences with extreme conservation between humans and rodents. We generalized their definition in a natural way that allows one to look for such extreme conservation over a much larger collection of species. We presented a linear time algorithm (Theorem 1) to find all such maximal length Extremely Conserved Elements, provided that one insists on conservation across all the aligned species. In contrast, we showed that, for the general case of conservation across an arbitrary subset of the species, the question of whether there is an Extremely Conserved Element is NPcomplete (Theorem 4), so it is unlikely an efficient algorithm exists for this general case [8]. This is true even in the special case of a gapless multiple sequence alignment.

Finally, we illustrated the linear time algorithm by identifying all Extremely Conserved Elements with $s=$ $40, t=100$, and $c=0.8$ in the currently available 44-vertebrate whole-genome alignment. The resulting $177 \mathrm{EC}(40,100,0.8)$ elements demonstrate many of the characteristics of the original ultraconserved elements [1].

\section{Methods}

In this section we describe two novel filters that make the application of Theorem 1 to whole-genome alignments feasible. Whole-genome MULTIZ alignments are divided into "alignment blocks" (Blanchette et al. [17]), where each block contains some subset of the species aligned to some region of the reference genome (human, in our case). A common reason for a boundary between alignment blocks is that some species enters or leaves the alignment at that boundary point. Our first filter simply reads through all the alignment blocks, retaining only those containing at least 40 species.

In theory it is possible that an $\mathrm{EC}(40,100,0.8)$ element could span an arbitrary alignment block $B$ consisting of $b$ columns that was discarded by Filter 1 , if there were enough perfectly conserved columns among 40 species before and after $B$ to make up for $b$ unconserved columns. However, if $b \geq 50$, this would mean that there is also an $\operatorname{EC}(40,100,0.8)$ element on one side of $B$ or the other. Therefore, we next merge alignment blocks as long as the separation between those blocks on the human genome is less than $50 \mathrm{bp}$; a separation greater than this signals the end of the current merged block and the beginning of a new one. More specifically, if $A$ is the current merged block, $C$ is the next alignment block following $A$ that was retained after Filter 1 , and $A$ and $C$ are separated by human sequence $B$ with $|B|<50$, we append $B C$ to the end of $A$ and insert the gap character "-" in row $s$ of any alignment column of $B$ or $C$ that was missing species $s$. 
Table 2: Effectiveness of the algorithmic filters in reducing the number of alignment columns to process

\begin{tabular}{lccc}
\hline & Filter I & Filter 2 & $\begin{array}{c}\text { Exhaustive } \\
\text { algorithm }\end{array}$ \\
\hline Columns to process & $3,843,856,747$ & $9,606,738$ & 220,646 \\
Time (in hours) & 2.6 & 0.1 & 47.3 \\
\hline
\end{tabular}

The running time of each phase of the algorithm (run on an Intel Xeon Quad Core X7350 $2.93 \mathrm{GHz}$ with I32 GB RAM) is also shown.

We are now in a position to describe Filter 2, which is run on each merged block independently. For the $i$-th alignment column of the current merged block, let $q_{i}=$ 1 if column $i$ has 40 or more equal characters (including "- "), and $q_{i}=0$ otherwise. Run the algorithm of Theorem 2 on the resulting string $q$ and $c=0.8$ to identify the maximum length substring $d$ of $q$ that contains at least $80 \% 1$ 's. Such a substring may not correspond to an EC $(40,100,0.8)$ element, because there is no assurance that the set of 40 species is the same for each column, but any $\mathrm{EC}(40,100,0.8)$ element must be contained in such a substring of length at least 100 . Filter 2, therefore, discards any merged block for which $|d|<100$.

On each merged block remaining after Filter 2, we now exhaustively try all combinations of at least 40 species and run the algorithm of Theorem 1 on each combination. In order to maximize the number of species in each $\mathrm{EC}(40,100,0.8)$ element, we enumerate the combinations in decreasing order from 44 species down to 40 species. Whenever we find an $\operatorname{EC}(40,100,0.8)$ element, we remove the longest such element for the current combination of species, and then run Filter 2 again on each of the two remaining pieces of the merged block, discarding either such piece if it fails to pass Filter 2. Note that this may sacrifice a longer $\operatorname{EC}(40,100,0.8)$ element in preference for one with a greater number of species if these elements overlap.

Table 2 shows the effectiveness of the two filters and the total running time of each of the three phases of the algorithm. Filter 1 removed all but $0.25 \%$ of its alignment columns and, of the remaining columns, Filter 2 removed all but $2.3 \%$. Since the full exhaustive algorithm on these remaining 220,646 alignment columns still required 47 hours of computing time, it is clear that the two filters are imperative.

The source code was written in Python and is available from the authors.

\section{Authors' contributions}

Both authors contributed equally and read and approved the final manuscript.

\section{Additional material}

\author{
Additional file 1 \\ EC40_100_80. A spreadsheet showing each EC(40, 100, 0.8) \\ element's human genome coordinates, its length, percent identity, \\ number of conserved species, names of missing species, name of the \\ human gene in which it resides (if appropriate), names of and distances \\ to the two nearest neighboring genes, and overlap with previously \\ identified conserved elements [146]. \\ Click here for file \\ [http://www.biomedcentral.com/content/supplementary/1471- \\ 2105-10-432-S1.XLSX] \\ Additional file 2 \\ EC40_100_80. An alignment file showing the exact alignment for each \\ $E C(40,100,0.8)$ element. \\ Click here for file \\ [http://www.biomedcentral.com/content/supplementary/1471- \\ 2105-10-432-S2.TXT]

\section{Additional file 3} \\ EC44_70_90. An alignment file showing the single longest extremely \\ conserved element that has at least $90 \%$ of its columns perfectly \\ conserved across all 44 vertebrate species. \\ Click here for file \\ [http://www.biomedcentral.com/content/supplementary/1471- \\ 2105-10-432-S3.TXT]
}

\section{Acknowledgements}

We thank Benno Schwikowski, Eddie Grove, Phil Green, Allan Borodin, and the students of CSE 427 for contributions and helpful discussions. We thank the anonymous reviewers for careful reading and suggestions that greatly improved the paper.

\section{References}

I. Bejerano G, Pheasant M, Makunin O, Stephen S, Kent WJ, Mattick JS and Haussler D: Ultraconserved elements in the human genome. Science 2004, 304:132I-1325.

2. Derti A, Roth FP, Church GM and Wu C-t: Mammalian ultraconserved elements are strongly depleted among segmental duplications and copy number variants. Nature Genetics 2006, 38:1216-1220.

3. Sakuraba $Y$, Kimura $T$, Masuya $H$, Noguchi $H$, Sezutsu $H$, Takahasi KR, Toyoda A, Fukumura R, Murata T, Sakaki Y, Yamamura M, Wakana S, Noda T, Shiroishi T and Gondo $Y$ : Identification and characterization of new long conserved noncoding sequences in vertebrates. Mammalian Genome 2008, 19:703-712.

4. Visel A, Prabhakar S, Akiyama JA, Shoukry M, Lewis KD, Holt A, Plajzer-Frick I, Afzal V, Rubin EM and Pennacchio LA: Ultraconservation identifies a small subset of extremely constrained developmental enhancers. Nature Genetics 2008, 40: I58-160.

5. Prabhakar S, Poulin F, Shoukry M, Afzal V, Rubin EM, Couronne O and Pennacchio LA: Close sequence comparisons are sufficient to identify human cis-regulatory elements. Genome Research 2006, 16:855-863.

6. Siepel A, Bejerano G, Pedersen JS, Hinrichs AS, Hou M, Rosenbloom K, Clawson H, Spieth J, Hillier LW, Richards S, Weinstock GM, Wilson RK, Gibbs RA, Kent WJ, Miller W and Haussler D: Evolutionarily conserved elements in vertebrate, insect, worm, and yeast genomes. Genome Research 2005, 15:1034-1050.

7. Kent WJ, Sugnet CW, Furey TS, Roskin KM, Pringle TH, Zahler AM and Haussler D: The human genome browser at UCSC. Genome Research 2002, 12:996-1006. 
8. Garey M and Johnson D: Computers and Intractability. A Guide to the Theory of NP-Completeness San Francisco: Freemann; 1979.

9. Allison L: Longest biased interval and longest non-negative sum interval. Bioinformatics 2003, 19:1294-1295

10. Chen KY and Chao KM: Optimal algorithms for locating the longest and shortest segments satisfying a sum or an average constraint. Information Processing Letters 2005, 96: | $97-20 \mid$.

I I. Wang $L$ and $X u$ Y: SEGID: Identifying interesting segments in (multiple) sequence alignments. Bioinformatics 2003, 19: 297-298.

12. Chung KM and Lu HI: An optimal algorithm for the maximumdensity segment problem. SIAM J Computing 2005, 34:373-387.

13. Goldwasser $M H$, Kao MY and Lu HI: Linear-time algorithms for computing maximum-density sequence segments with bioinformatics applications. I Computer and System Sciences 2005, 70:128-144.

14. Johnson D: The NP-completeness column: an ongoing guide. Jlgorithms 1987, 8:438-448.

I5. Zhang B, Kirov S and Snoddy J: WebGestalt: an integrated system for exploring gene sets in various biological contexts. Nucleic Acids Research 2005, 33:W74I-748.

16. Oin Z, Ren F, Xu X, Ren Y, Li H, Wang $Y$, Zhai $Y$ and Chang $Z$ ZNF536, a novel zinc finger protein specifically expressed in the brain, negatively regulates neuron differentiation by repressing retinoic acid-induced gene transcription. Molecular and Cellular Biology 2009, 29:3633-3643.

17. Blanchette M, Kent WJ, Riemer C, Elnitski L, Smit AF, Roskin KM, Baertsch R, Rosenbloom K, Clawson H, Green ED, Haussler D and Miller W: Aligning multiple genomic sequences with the threaded blockset aligner. Genome Research 2004, 14:708-7I5.

Publish with BioMed Central and every scientist can read your work free of charge

"BioMed Central will be the most significant development for disseminating the results of biomedical research in our lifetime. "

Sir Paul Nurse, Cancer Research UK

Your research papers will be:

- available free of charge to the entire biomedical community

- peer reviewed and published immediately upon acceptance

- cited in PubMed and archived on PubMed Central

- yours - you keep the copyright

Submit your manuscript here:

http://www.biomedcentral.com/info/publishing_adv.asp
BioMedcentral 